Cinémas

Revue d'études cinématographiques

Journal of Film Studies

\title{
L’Erreur, l'errance : deux visions de la géographie américaine
}

\section{François Jost}

Volume 1, numéro 1-2, automne 1990

Américanité et cinéma

URI : https://id.erudit.org/iderudit/1000988ar

DOI : https://doi.org/10.7202/1000988ar

Aller au sommaire du numéro

Éditeur(s)

Cinémas

ISSN

1181-6945 (imprimé)

1705-6500 (numérique)

Découvrir la revue

Citer cet article

Jost, F. (1990). L’Erreur, l'errance : deux visions de la géographie américaine. Cinémas, 1(1-2), 20-30. https://doi.org/10.7202/1000988ar

\section{Résumé de l'article}

L'auteur étudie la signification de la présence d'une américanité à travers quelques films du cinéaste allemand Wim Wenders. L'américanité y apparaît à travers un modèle de récit selon lequel l'histoire signifie avant tout scénario, enchaînement d'actions, et utilisation de la ville-espace. Mais ce modèle est confronté, chez le cinéaste, à une volonté d'inscrire un récit premier, de refonder une temporalité, d'inscrire une conception de la ville-temps. Le concept d'américanité apparaît alors selon une dualité de points de vue et ne renvoie pas à l'origine continentale du réalisateur. 


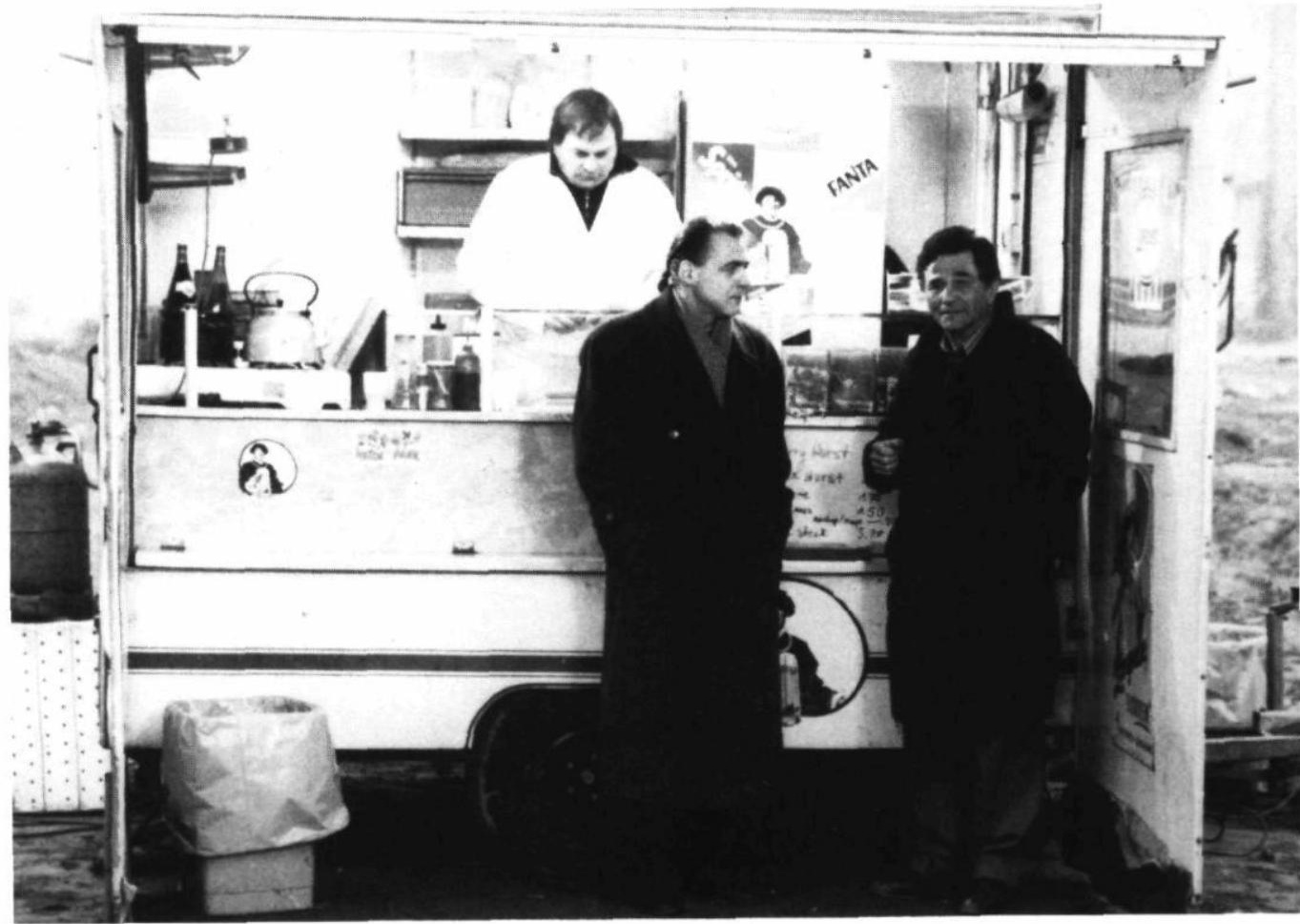

Les Ailes du désir de Wim Wenders (1987)

Collection Cinémathèque québécoise 


\title{
L'Erreur, l'errance: deux visions de la géographie américaine
}

\section{François Jost}

\begin{abstract}
RÉSUMÉ
L'auteur étudie la signification de la présence d'une américanité à travers quelques films du cinéaste allemand Wim Wenders. L'américanité y apparaît à travers un modèle de récit selon lequel l'histoire signifie avant tout scénario, enchaînement d'actions, et utilisation de la ville-espace. Mais ce modèle est confronté, chez le cinéaste, à une volonté d'inscrire un récit premier, de refonder une temporalité, d'inscrire une conception de la ville-temps. Le concept d'américanité apparaît alors selon une dualité de points de vue et ne renvoie pas à l'origine continentale du réalisateur.
\end{abstract}

\begin{abstract}
The author studies the significance of the presence of a certain "Americanness" in several of the films of German filmmaker Wim Wenders. Americanness can be found in a narrative model in which the story has meaning above and beyond the scenario, the sequence of actions, and the use of city-space, but which confronts the filmmaker's desire to tell a primary story, to recreate temporality, to inscribe a conception of city-time. Thus the concept of Americanness emerges in a duality of points of view and has nothing to do with the director's continent of origin.
\end{abstract}

Comment ne pas penser à Roland Barthes en prononçant ce mot américanité? Comment ne pas évoquer ce mode de dérivation linguistique qu'il proposait, dès 1957, pour nommer certains concepts? Il écrivit à ce sujet: 
La Chine est une chose, l'idée que pouvait s'en faire, il n'y a pas longtemps encore, un petit-bourgeois français en est une autre; pour ce mélange spécial de clochettes, de pousse-pousse et de fumeries d'opium, pas d'autres mots possibles que celui de sinité. Cela n'est pas beau? Que l'on se console au moins en reconnaissant que le néologisme conceptuel n'est jamais arbitraire; il est construit sur une règle proportionnelle fort sensée (p. 206).

Et Barthes d'ajouter en note latin/latinité $=$ basque $/ \mathrm{x} \times \mathrm{x}=$ basquité. Si ce rappel me semble nécessaire, c'est que le formidable succès de ce mode de construction des néologismes nous fait souvent oublier l'un des deux sèmes que le texte que je viens de citer met en valeur. Le suffixe «ité» ne définit pas seulement un concept, une essence (la latinité, c'est le propre du latin), il renvoie aussi à l'idée que l'on peut se faire de cette essence. Autrement dit: il ne caractérise pas seulement une entité abstraite, une idée platonicienne, mais la représentation sociale et ethnique qu'on en a («l'idée de la Chine d'un petit-bourgeois français»).

$\mathrm{Ce}$ que je voudrais esquisser, c'est précisément comment le concept d'américanité est l'objet d'une dualité de points de vue, de visions, qui ne renvoie pas seulement à l'origine continentale du réalisateur, mais qui est solidaire d'une opposition plus profonde entre un mode de narration généralisé, le cinéma classique hollywoodien, tel qu'il a pu être défini par Bordwell, et un mode de narration plus restreint, plus idiolectal, sans doute plus européen, comme celui que donnent à voir les films de Wenders. La question ultime étant: pourquoi un cinéaste comme Wenders a-t-il besoin d'une certaine américanité pour faire un cinéma allemand? Et quelle idée de l'Amérique lui est nécessaire?

Dans Les Ailes du désir (1987) sont proposées deux «figures» du récit: d'un côté Colombo qui est la figure emblématique des histoires (il n'est d'histoire que policière); de l'autre, l'ange qui veut entrer dans la temporalité, dire maintenant et pas toujours, ne pas tout savoir, qui veut aussi «se conquérir une histoire», commencer par «il était une fois et il sera».

D'un côté, donc, un modèle américain, incarné par Peter Falk, selon lequel l'histoire signifie avant tout scénario, enchaînements d'actions, conflit; de l'autre, un modèle proposé par un ange allemand, déçu et bientôt déchu, dans ce «pays du récit» qu'est l'Allemagne selon l'un des personnages du film. Pays du récit, si, comme le dit Jean-Luc Nancy, le récit premier est à la fois l'histoire du commencement du monde ou du commencement du récit lui-même ${ }^{1}$.

Comment ne pas voir dans cette allusion au récit originel sur fond de ville en ruines et de mur-frontière, dans cette définition de l'histoire comme pure temporalité, et non comme suite 
d'événements, une tentative, presque explicitement formulée, pour assumer la démarcation, la séparation, en somme pour assumer Berlin, ville amnésique de son origine, c'est-à-dire de sa fondation, cet acte, comme le note Michel Serres, par lequel «la multiplicité se fait unité» (p. 119) et le consilium individuel, «consensus». Mais que vient faire Peter Falk, l'acteur américain, Colombo, le policier, dans ce pays et dans ce scénario? La fusion de l'Histoire du cinéma, lisible dans cette thèse dont un jeune Allemand se fait l'écho auprès de Falk, et selon laquelle Hitler n'aurait été qu'un acteur payé par Goebbels, nous fournit une piste: la réponse est peut-être bien dans la confrontation d'une Amérique scénarisée, héritage d'Hollywood, et dans une vision rêvée d'un pays réel, telle qu'elle se donne à lire dans quelques films de Wenders.

Qu'est-ce en effet que la ville dans le film hollywoodien? Si la fondation peut y jouer comme point d'arrivée, cet ordre philosophique qui repose sur une harmonie des parties dans le respect mutuel de l'organisation de l'urbs, est par essence étranger à l'idée même de scénario. Tout simplement parce qu'une ville fondée, et qui ne bougerait plus de cet état, serait une ville sans histoire, à la lettre: une ville sans scénario, puisqu'elle ne permettrait aucun conflit. La démarcation produite par l'urbanisation, et non par la ville, est l'un des paramètres qui permet l'écriture d'un récit hollywoodien, parce qu'il aide à mobiliser ces autres règles, recensées par Bordwell, que sont la définition du personnage par son occupation, son âge, mais surtout son «ethnic identity», la naissance d'un conflit à partir des désirs et des aspirations de ce personnage, et la contrariété de deux lignes d'action, dont l'une est souvent constituée par une histoire d'amour ${ }^{2}$.

Comme modèle de cette utilisation de la ville-espace, à l'opposé de la ville-temps qui arrête son cours pour refonder sa temporalité, j'ai choisi un film de série B: East Side, West Side (Ville haute, ville basse, M. Le Roy, 1949). Le film s'ouvre sur une vue panoramique de New York. En même temps, on peut entendre la voix over de Jessie, l'héroïne du film:

Voici ma ville. Vous la connaissez à travers des films. On dit que c'est la ville la plus excitante, la plus angoissante, la plus rapide. On a tant dit sur son rythme fou, le battement de son coeur. Son rythme est celui de ma vie. Son battement de coeur, c'est le mien.

L'homologie de la ville et de l'organe n'est plus comme dans la philosophie grecque et comme dans Metropolis, la métaphore d'un mythe de la fondation. Ce qu'annonce cette voix, dès les premières images, c'est que la constitution du personnage, sa caractérisation reposent sur l'identification symbolique au tissu urbain. Que le personnage soit à New York ou non, peu importe: New York est en elle (comme Manhattan est en W. Allen). Dans ce type de film, 
la ville américaine peut presque disparaître comme décor représenté: même quand l'action se déroule dans des lieux clos, le découpage urbain est encore au premier plan, parce qu'il est intériorisé par les personnages, qui en portent toujours la marque (dans nombre de feuilletons américains au titre toponymique, «l'action» se déroule majoritairement en intérieur). La ville n'est pas l'urbs, à l'image de cet être humain idéal, où chaque organe accomplit sa fonction, c'est la ville dans son développement, dans son urbanisation, qui modèle l'individu à son image.

Voyons comment ce découpage fonde le scénario. Quand le film commence, un couple se remet tout juste d'une crise qu'il vient de traverser en raison de la liaison du mari, Bran, avec une autre femme, Isabel. Celle-ci, que l'on croyait éloignée, reparaît un soir dans une boîte de nuit où Bran est allé sans sa femme. Une bagarre éclate. Bran est jeté à terre. À la suite de circonstances qu'il serait fastidieux d'énumérer, Rosa présente son ami Mark à la femme de Bran, Jess. Un regard, une rencontre. Ce qui est posé dès le départ, c'est la différence de statut social: Mark, d'abord petit flic d'origine italienne, est maintenant membre éminent de l'«intelligence service». Jess, quant à elle, appartient à la rubrique mondaine, sans que l'on connaisse bien son passé. D'un côté, donc, un personnage qui est défini par une «trajectoire ascendante et verticale», pourrait-on dire dans les termes de Bourdieu, trajectoire qui est un topos de l'américanité, notamment dans les films où les personnages doivent être goal-oriented; de l'autre, une femme caractérisée par le quartier où elle vit (Grammercy Park). Dès lors, deux logiques sont à l'œuvre dans le scénario: celle de l'identification (Mark est encore, et toujours, West side) et celle de l'assignation sociale (Jess habite East side).

À celui qui est né West side, il n'est pas possible de pénétrer East side sans difficulté: la frontière implicite qui sépare les deux personnages est manifeste dès la première scène où Mark accompagne Jess chez elle. Avant de franchir la porte de son appartement de Grammercy Park, elle souligne la valeur symbolique de ce geste pourtant anodin: «Je m'excuse, vous venez de quitter la misère.»

Un peu plus tard, c'est Jess qui accompagne Mark dans son quartier d'origine, la Petite Italie: il assortit cette visite du commentaire suivant: «Toujours dans votre beau quartier, vous ne connaissez pas le mien. C'est à des millions de kilomètres.» La proximité spatiale dans la ville ne rapproche donc nullement les habitants dès lors que l'urbanisation a tracé une frontière symbolique. Pourquoi? Mark répond à sa façon: «Chacun voit avec ses yeux. J'écris ce que je vois.» «Si vous savez écrire», réplique son éditeur. Et Jess d'ajouter: «Il a commencé en écrivant des contra- 
ventions.» Il n'existe donc plus dans cette ville un regard unificateur. L'espace de la ville équivaut à l'espace social. On a le regard de son quartier, mais aussi celui de son habitus (Bourdieu).

Seul l'amour permettrait l'osmose des deux régions délimitées. D'où le conflit inhérent à de tels scénarios: comment transformer la rencontre en histoire d'amour, quand l'espace intériorisé par les personnages les en écarte? La question est formulée d'ailleurs par une réplique de Mark: "Quand on s'aime, la géographie n'y peut rien, mais aucune chance d'être ensemble. Nos vies passent par des chemins différents.» Comme on le voit, le présent de l'urbain, ancré dans la ville américaine réelle, souffre d'une impossibilité de se refonder. Il n'y a qu'une seule issue: que l'homme et la femme aient la même identification urbaine. Comme on l'aura deviné, c'est précisément ce que l'avancée du film révélera. Au détour d'une rue de West side, Jess désigne le lieu de sa naissance: «Vous voyez cette maison, je suis née dedans.» Et Mark de lui répondre: «Voilà pourquoi je me sens si bien avec vous, je vous connais si bien et si peu.»

Le vrai couple n'est donc pas celui qu'a constitué le mariage, mais celui qu'a formé le quartier: la ville de la mère, de la naissance (non plus la metropolis, la ville-mère), celle dont le découpage est intériorisé par le personnage, est plus forte que celle à laquelle a abouti la trajectoire sociale. La loi de cette ville est «qui se rassemble se ressemble». Cette origine est une erreur qui pèse sur le scénario à la manière d'une fatalité, dans la mesure où elle détermine l'amour par identification de son personnage à son passé. Il faudrait faire cette géographie de l'erreur dans le cinéma américain qui recenserait les différentes frontières possibles à l'intérieur de la multiplicité des tissus urbains. La ville intériorisée peut en effet prendre des allures différentes selon qu'on a affaire à un policier, à un drame psychologique ou à un western. Dans tous les cas, elle est une fatalité qui pèse sur le monde diégétique et qui fait surgir le conflit narratif dans l'opposition entre être (appartenance à la ville intériorisée) et paraître (jeu social). Dans une certaine mesure, le scénario doit s'efforcer de nous faire découvrir un monde, un microcosme dans un macrocosme. C'est encore la logique de Colombo.

La disparition de la frontière, c'est la disparition de la mère, la disparition du scénario hollywoodien classique: c'est cette corrélation que donne à lire la vision européenne de l'Amérique à l'œuvre dans certains films de Wenders. Considérons d'abord Alice dans les villes (1974). Une sorte de journaliste, Philippe, parcourt l'Amérique pour un reportage sur le paysage, qu'il ne réussit pas à décrire. Obligé de rentrer en Allemagne, il rencontre devant le guichet d'une compagnie aérienne une femme et sa petite fille, 
Alice. Celle-ci étant abandonnée par sa mère, il va entreprendre avec elle une longue dérive: de New York en Hollande, de ville en ville, à la recherche d'une hypothétique grand-mère.

Qu'apprend-on sur les villes que l'on traverse? Rien. Rien en tout cas qui les distingue les unes des autres. Pas le moindre élément pittoresque qui différencierait un tissu urbain d'un autre. La figure privilégiée est le travelling et les seuls points d'arrêt sont les bars et les hôtels. Loin des aires aux frontières bien distinctes, les villes sont un espace continu, un continuum indifférencié, à l'image de ce «paysage américain» dont Philippe dit: «À partir du moment où on quitte New York, tout est pareil, tout se ressemble au point qu'on ne réussit plus à imaginer un changement. Je suis devenu étranger pour moi-même.» En d'autres termes, il n'y a plus d'identification à un quelconque lieu. Cette vision de l'Amérique est-elle européenne? En tout cas, force est de constater que c'est aussi celle de Baudrillard qui écrit:

\begin{abstract}
À Paris, le ciel ne décolle jamais, il ne plane pas, il est pris dans le décor des immeubles souffreteux qui se font de l'ombre les uns aux autres, comme la petite propriété privée — au lieu d'être la façade miroir vertigineuse, comme celle du grand capital à New York. Ça se voit au ciel: l'Europe n'a jamais été un continent. Dès que vous posez le pied en Amérique du Nord, vous sentez la présence d'un continent entier - l'espace y est la pensée même (p. 22).
\end{abstract}

Comment ne pas voir dans ce retour du même une sorte de paradis qui permet à l'Européen de naviguer dans un espace divisé, mais dépassionné? L'impossibilité de Philippe d'écrire sur le paysage américain est l'impossibilité de découper l'univers traversé. La ville est à la surface et la photo est la seule preuve que son «moi existe encore». Le cogito est vidé de son identité, réduit à un percipi désincarné: «une preuve que tu as été ça» dit une jeune femme à Philippe: «celui qui a vu». Or Baudrillard a formulé ce que celui-ci ne réussit pas à dire et que Wenders nous montre à travers l'errance de son personnage. L'Amérique «exorcise la question de l'origine $(\ldots)$, elle n'a pas de passé ni de vérité fondatrice (..). Elle vit dans l'actualité perpétuelle (...). Elle n'a pas de problème d'identité. (...) C'est l'utopie réalisée» (p. 76). Il ajoute: «Notre conception de la liberté ne pourra jamais rivaliser avec la leur, spatiale et mobile, qui découle du fait qu'ils se sont un jour affranchis de cette centralité historique» (p. 80). Cette phrase amène l'essayiste à cette conclusion: «Les Êtats-Unis sont un paradis» (p. 96).

On comprend, dans ces conditions, que le passage par l'Amérique soit pour Wenders un passage obligé, non une simple fascination, dont l'effet cathartique est de permettre d'assumer 
I'Europe, comme s'il retournait cette autre proposition de Baudrillard selon laquelle pour voir et sentir l'Amérique, il faut s'être demandé «comment peut-on être européen?» (p. 102). Cette régression vers l'origine, celle d'Alice en l'occurrence, peut alors s'effectuer dans l'indifférence de cette liberté errante, sans qu'aucun indice d'ordre affectif n'ordonne le monde. Seule l'énumération alphabétique des villes allemandes guidera plus ou moins la petite fille dans sa quête. La crise d'identité que traversent les deux personnages, repérable entre autres dans la perte d'identification de l'origine, est d'abord la crise d'identité de la ville: Alice, abandonnée par la mère, abandonne, à sa façon, la ville-mère. L'errance dans le paysage américain est un modèle rassurant, qui permet de mettre à distance l'idée européenne d'une fondation consensuelle de la ville par l'ensemble de ses habitants. Très curieusement, cette difficulté à distinguer entre les villes, entre la ville et la nature, cette perte de la frontière, finit par «japoniser»l'espace américain, au sens où l'espace japonais privilégie, à l'inverse de l'espace frontière que nous connaissons, les zones intermédiaires qui, comme dit $\mathrm{A}$. Berque, «constitue[nt] un passage obligé, un sas ou un tampon», comme ces engawa qui se trouvent aux confins de l'intérieur et de l'extérieur de la maison «où l'on est ni dehors ni dedans.» D'où la profusion, chez Wenders, des lieux intermédiaires, portes à tambour, motels aux confins de la ville ou de l'aéroport, «snacks» au bord de route, etc. Ce n'est d'ailleurs pas un hasard si l'alternative à l'Amérique est le Japon autant pour Wenders (Tokyo-Ga et $\mathrm{Ozu}$ ) que pour Bordwell.

Chez Wenders la disparition du personnage, sa perte d'identité, est solidaire de l'uniformisation géographique, de la perte d'un espace qualifié (où l'on distingue le West side du East side). À ce titre, Paris, Texas (1984) pousse Alice à son paroxysme. «Pas de désir: le désert» (p. 119). Cette formule empruntée à Baudrillard, n'est-elle pas comme l'alpha de Travis? L'identité perdue, enfouie dans les limbes de la mémoire abandonnée, la perte de la faculté de nommer ont cédé la place à une indifférence généralisée. Après l'abandon de la mère, sa quête. L'oméga du désir de Travis sera l'origine sous sa double figure: celle de la mère - la sienne propre, celle de son enfant - , et le lieu de la naissance: ce terrain sans aspérité où «son père et sa mère ont fait l'amour» («ma vie a commencé là-bas», dit Travis), terrain dont le nom renvoie de façon emblématique à l'Europe. L'oméga de l'errance, c'est la ville et la mère, réunies dans ce moment où il comprend qu'il est aussi un père, au moment où, en quelque sorte, il est resitué dans le temps, dans un avant et un après, en bref: dans l'histoire. Le détour par l'Amérique, c'était «se perdre dans un pays où, dit Travis, il serait complètement anonyme, dans un pays sans langage», vivre 
l'étrangeté, l'«extranéité», qui n'est rien d'autre que l'absence à son propre monde: «Ça fait longtemps que je suis absent?» demande-til à son frère. "Quatre ans», répond ce dernier. "Ça fait longtemps quatre ans?» ajoute Travis.

Si l'Amérique est un paradis perdu, un pur espace continu et indifférencié où le temps n'est que déplacement, errance, il faut pour vivre l'Europe quitter un jour cette vision angélique, cette absence au monde, retrouver le lieu de la naissance pour naître à nouveau. C'est bien le sens de la décision d'Ariel dans Les Ailes du désir, qui répond comme un écho à l'errance hallucinée de Travis: «À moi de me conquérir une histoire (...). J'ai été assez longtemps absent, hors du monde.»

On comprend mieux maintenant pourquoi le personnage joué par Peter Falk, cet ex-ange, est l'agent de cette transformation. Avec Les Ailes du désir, on abandonne l'Amérique rêvée, l'Utopie réalisée. On est chassé du paradis. De cette métaphore, Wenders fait un film. Or Peter Falk, avec tout ce qu'il représente d'histoires, de scénarios de la limite et de la frontière, de la démarcation, peut véhiculer cette vision de l'Amérique non plus idéale ou idéologique, mais forgée dans et par le cinéma, cette Amérique scénarisée, à mille lieux du fantasme européen. Et il apparaît au terme de ces pensées de l'ange qui se rend sur les lieux de son tournage:

Y a-t-il encore des frontières? Plus que jamais. Chaque rue a sa propre frontière (...). Chaque maître de maison cloue son nom sur la porte et étudie le journal comme un maître du monde. Le peuple allemand a éclaté en autant de petits états qu'il y a d'individus.

À l'éternité indéfinie du toujours il substitue la temporalité du maintenant; à l'espace indifférencié, le territoire. Aussi, dans le Berlin des Ailes du désir, il n'y a plus d'étendues à perte de vue, plus de lieux intermédiaires, d'engawa. Le mur est toujours là, les lieux sont «qualifiés», socialisés, compartimentés en vue d'activités précises: travail, lecture, déplacement ou plaisir. À Alice qui cherchait le lieu de son origine par ordre alphabétique répond la quête d'un vieil homme cherchant à retrouver Postdamer Platz, sous et malgré les décombres. L'univers spatial n'est plus amnésique: il est dans la durée. À la vision en surplomb d'Alice ou de Paris, Texas qui commence, comme on sait par une plongée sur le désert, une vue divine sur le néant, se substitue une vision à hauteur d'homme, en un sens caractéristique du cinéma de fiction classique: «En avant dans le gué du temps, le gué de la mort, dit l'ange. Regarder n'est pas regarder d'en haut, mais à hauteur d'œil.» 
N'était-ce pas le constat du héros de l'urbanisation? «Chacun voit avec ses yeux.» Ce que Peter Falk apporte finalement, via le scénario hollywoodien, c'est une vue à hauteur d'homme constitutive de l'espace de la ville, en même temps que l'amour comme topos de la réconciliation sociale.

C'est nous qui sommes le temps à présent (...). Pas seulement la ville entière, le monde entier prend part à notre décision. Nous deux sommes plus que deux, désormais, nous incarnons quelque chose. Nous voilà sur la place du peuple et toute la place est pleine de gens. Nous déterminons le jeu pour tous. Il n'y a pas plus grande histoire que la nôtre. Celle de l'homme et de la femme.

Contrairement à Metropolis, qui refondait la ville sur le consensus social, Wenders propose une fondation anthropologique, l'individu ayant valeur d'humanité universelle comme chez Rousseau. Le microcosme de l'histoire amoureuse vaut comme métaphore de l'histoire: un homme, une femme, «de nouveaux ancêtres», «il sera une fois». L'injection du modèle américain scénarisé, sous forme emblématique, parallèle de l'abandon d'une géographie imaginaire de l'Amérique, refonde à la fois l'Histoire avec un grand $H$, le Temps, et l'histoire avec un petit $h$, l'événement, ces deux histoires que nous opposions au début. Fonder le scénario sur des transformations et non sur la simple répétition du même, introduire de l'erreur dans l'errance (la trapéziste qui a mal choisi son cirque, par exemple) remet le temps en route.

Au grand rêve européen de la cité refondée par la volonté de tous, rêve un jour oublié dans l'espace sans limite d'une Amérique fantasmée, se substitue l'amour réparateur, seul capable de remettre le temps en route, d'assumer la démarcation spatiale. Dans cette perte de l'«atopie» et de l'utopie, il y a pourtant un gain: le récit. N'est-ce pas ce que Wenders voulait dire quand, après Paris, Texas, il déclarait que, dorénavant, il savait raconter des histoires?

Université de Paris III

\section{NOTES}

1 Voir à ce sujet Jean-Luc Nancy, La Communauté désaeuvrée (Paris: Christian Bourgois, 1990).

2 Voir à ce sujet D. Bordwell, J. Staiger, K. Thompson, The Classical Hollywood Cinema (New York: Columbia University Press, 1985).

\section{OUVRAGES CITÉS}

Barthes, Roland. «Le Mythe aujourd'hui», Mythologies. Paris: Seuil, 1957. 
Baudrillard, Jean. Amérique. Paris: Grasset, 1986. Ouvrage repris dans la collection «biblio essais» du Livre de poche, 1988. Toutes les citations renvoient à cette édition.

Berque, A. Vivre l'espace au Japon. Paris: PUF, 1982.

Serres, Michel. Rome, le livre des fondations. Paris: Grasset, 1983. 